\title{
Aproximación clínica y principales rickettsiosis transmitidas por garrapatas presentes en Latinoamérica
}

\author{
Katia Abarca y José A. Oteo
}

\section{Clinical approach and main tick-borne rickettsiosis present in Latin America}

Tick-borne rickettsial diseases are potentially life threatening infections that in Latin America have an emerging and reemerging character. Until few years ago, Rickettsia rickettsia was the only tick-borne rickettsia present in America; but nowadays several other species such as $R$. parkeri and $R$. massiliae are causing disease in humans in the region. In addition, new species are being described; although their pathogenicity has not been confirmed they should be considered as potential pathogens. Since the microbiological diagnosis of rickettsioses can take days or weeks, a high clinical suspicion and early start of appropriate treatment are crucial. In this review the distribution and main clinical manifestations of tick-borne rickettsial diseases in Latin America are detailed. Since $R$. felis has been found in ticks and the role of this vector has not been clarified, we have included a section about this pathogen.

Palabras clave: Rickettsiosis; Latinoamérica; garrapatas; fiebre exantemática, Rickettsia rickettsii; Rickettsia parkeri; Rickettsia massiliae; Rickettsia felis.

Key words: Rickettsioses; Latin America; ticks; spotted fever, Rickettsia rickettsii; Rickettsia parkeri; Rickettsia massiliae; Rickettsia felis.

\section{Introducción}

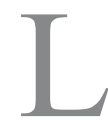

as rickettsiosis son afecciones de distribución mundial provocadas por diferentes especies de

Rickettsia. Son transmitidas por artrópodos vectores (piojos, pulgas, garrapatas duras y otras especies de ácaros) y por diferentes motivos se consideran infecciones emergentes y re-emergentes ${ }^{1}$. Su epidemiología ha estado siempre ligada a un círculo formado por la interacción reservorio -artrópodo vector- hombre, en el que juegan un papel importante las costumbres sociales, medio ambiente, situación geográfica e incluso la situación político-social. Las rickettsiosis han tenido gran trascendencia a lo largo de la historia, como es el caso del tifus exantemático que, si bien parece controlado, sigue siendo una amenaza en el contexto de guerras, desastres naturales o cualquier circunstancia que propicie falta de higiene o alteraciones medioambientales. Fruto de los avances y accesibilidad a los nuevos métodos de estudio y detección microbiológica, en los últimos años el número de especies de Rickettsia que han sido implicadas en patología humana se ha incrementado en gran medida, especialmente la descripción y número de rickettsiosis transmitidas por garrapatas duras ${ }^{2-4}$.

En Latinoamérica, a pesar de que existen muy buenas descripciones de estas afecciones en la primera mitad del siglo pasado, se puede decir que ha existido un vacío posterior en su estudio y descripción, hasta hace escasos años $^{2}$. Con el reporte de nuevos casos de infección por Rickettsia rickettsii en Centroamérica y otras zonas de Sudamérica después de muchos años sin su ocurrencia, y la emergencia de casos humanos provocados por otras especies de Rickettsia, se ha despertado un gran interés por estas infecciones. Dado que se trata de infecciones potencialmente letales, y que el no distinguir o sospechar estas afecciones puede conducir a la muerte, consideramos que una puesta al día de los signos y síntomas con los que se presentan los pacientes afectos de rickettsiosis puede ser de gran utilidad para los médicos clínicos. En esta revisión ofrecemos una revisión de las manifestaciones clínicas y una actualización de la distribución de las rickettsiosis transmitidas por garrapatas presentes en Latinoamérica.

\section{Manifestaciones clínicas de las infecciones por rickettsias transmitidas por garrapatas en Latinoamérica}

Los síntomas y signos clínicos de las rickettsiosis comienzan usualmente en 6 a 10 días posteriores de la picadura o exposición al artrópodo vector competente, e incluyen: fiebre, malestar general, cefalea, mialgias y diferentes tipos de lesiones cutáneas, que van desde un exantema maculo-papular o papulo-vesicular leve a cuadros petequiales intensos, acompañados o no de una escara de inoculación (Figuras 1, 2 y 3). El exantema suele
Pontificia Universidad Católica de Chile. Santiago, Chile. División de Pediatría, Escuela de Medicina (KA).

Hospital San Pedro-Centro de Investigación Biomédica de La Rioja. Logroño,España. Centro de Rickettsiosis y Enfermedades Transmitidas por Artrópodos Vectores. Departamento de Enfermedades Infecciosas (JAO).

Los autores declaran no tener conflicto de interés.

Fuente de financiamiento: no hubo.

Recibido: 22 de enero de 2014 Aceptado: 15 de mayo de 2014

Correspondencia a: Katia Abarca Villaseca katia@med.puc.cl; abarcakatia@ gmail.com 
Figura 1. Exantema maculopapular purpúrico en un paciente afecto de infección por Rickettsia rickettsii.
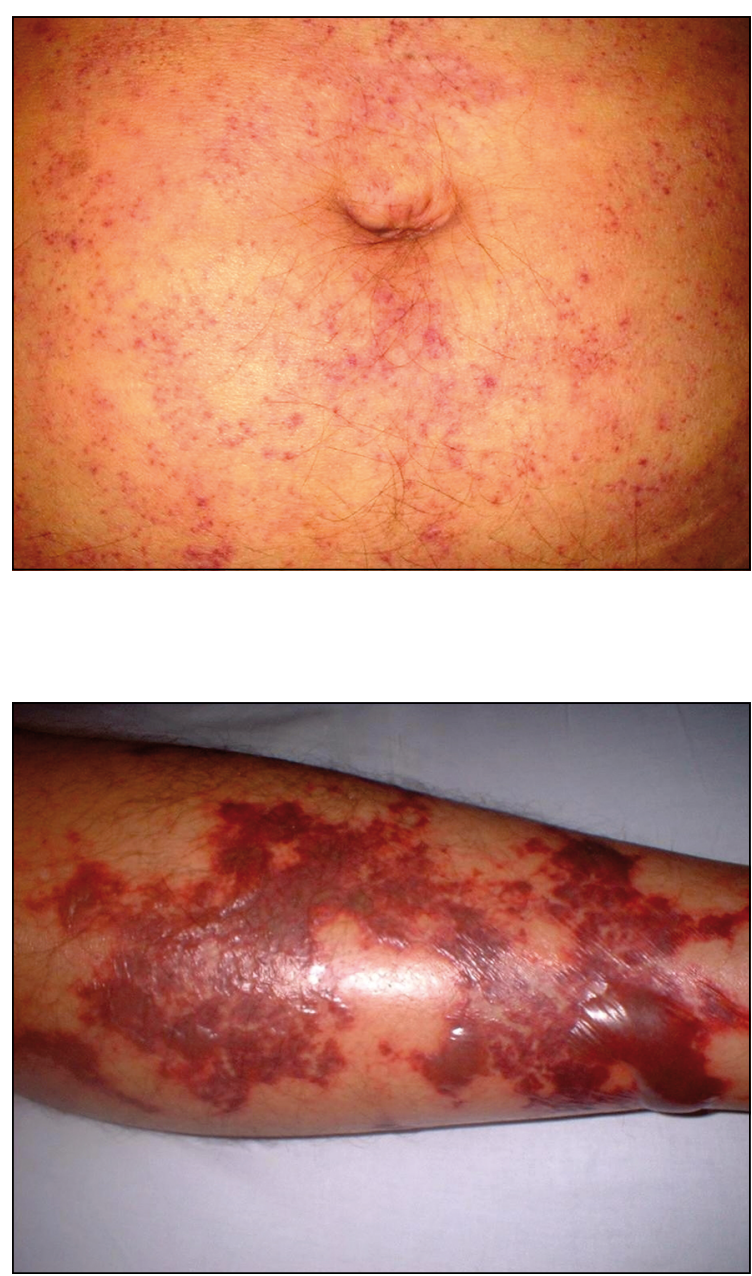

Figura 2. Equimosis extensa en paciente afecto de infección por Rickettsia rickettsii.

Figura 3. Escara y celulitis con exantema maculo-papular en paciente con infección por Rickettsia parkeri. comprometer palmas y plantas 5 . Estas manifestaciones clínicas y su gravedad están intimamente relacionadas con el mecanismo patogénico común a todas las rickettsiosis, que es la vasculitis de pequeño-mediano vaso. Todas las especies de Rickettsia no son igual de patógenas; así, mientras $R$. rickettsii puede provocar con frecuencia cuadros clínicos muy graves con alta mortalidad, $R$. parkeri provoca cuadros mucho más leves y de momento no se ha descrito mortalidad. El estado inmunológico del paciente y la respuesta inflamatoria mediada por diferentes citoquinas van a determinar, junto a los factores antes señalados, las manifestaciones clínicas y su gravedad. En los cuadros graves se puede observar compromiso orgánico del sistema nervioso central, pulmonar, cardíaco, renal, trastornos de la coagulación y finalmente falla multiorgánica 5 .

En los exámenes generales de laboratorio se puede encontrar trombocitopenia, leucopenia o leucocitosis, aumento de las transaminasas hepáticas y de reactantes de fase aguda como la proteína $\mathrm{C}$ reactiva-PCR entre otros, sin que exista ninguna determinación bioquímica o hematológica específica de rickettsiosis.

\section{Rickettsia rickettsii}

Es el agente productor de la fiebre manchada de las Montañas Rocosas, la más grave de las rickettsiosis transmitidas por garrapatas. Se han documentado casos humanos de $R$. rickettsii en los siguientes países de la región: Brasil, donde es conocida como fiebre manchada brasileña $^{6-10}$, México ${ }^{11-14}$, Panamá ${ }^{15-17}$, Costa Rica ${ }^{18-20}$, Colombia $^{21-26}$ y Argentina ${ }^{27,28}$. Muchos de estos casos se han producido como brotes familiares o en zonas geográficas cercanas.

Los síntomas suelen iniciarse entre 5 y 10 días después de la picadura de la garrapata, y dado que gran parte de estas picaduras pueden pasar desapercibidas, se debe tener un alto índice de sospecha. El cuadro clínico incluye fiebre elevada de aparición súbita, con escalofríos, malestar general, mialgias, artralgias, cefalea, vómitos, fotofobia y exantema. Aunque la fiebre y el exantema, en un adecuado ambiente epidemiológico, son los signos que deben hacer pensar en una rickettsiosis, la tríada de fiebre, exantema y antecedente de picadura de garrapata sólo está presente en $3 \%$ de los pacientes durante los primeros tres días $^{20}$. El exantema puede ser maculo-papular y a menudo petequial (Figura 1). Suele iniciarse en extremidades con afectación de palmas y plantas y progresar posteriormente al tronco; en ocasiones se presentan equimosis múlti-

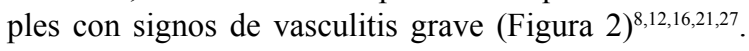
La frecuencia de exantema es variable en las distintas series, habiéndose descrito entre 18 y $100 \% \%^{8,12,13,20,23,25}$. Se han observado manifestaciones gastrointestinales, en especial en los niños, consistentes en náuseas, vómitos y dolor abdominal ${ }^{9,10,13,15,25,27}$. En un paciente se describió 
edema periorbitario con corioretinitis ${ }^{27}$. De forma muy excepcional, (solamente en dos comunicaciones en Latinoamérica), se ha documentado la presencia de escara en el sitio de la picadura y linfadenopatía regional ${ }^{11,17}$. En aproximadamente $25 \%$ de los casos se presentan síntomas de gravedad, como insuficiencia respiratoria, shock, ictericia, insuficiencia renal, efusión pericárdica y pleural, manifestaciones hemorrágicas que incluyen necrosis de dedos, ortejos o del pabellón auricular y compromiso neurológico en forma de convulsiones, disminución de la conciencia, parestesias, rigidez de extremidades y debilidad muscular ${ }^{8,10,12,13,15-17,21,24,27-29}$. En los casos de desenlace fatal, éste se produce por insuficiencia respiratoria o falla multiorgánica ${ }^{23,27,28}$.

En los exámenes de laboratorio se puede apreciar leucocitosis o leucopenia con desviación izquierda, trombocitopenia, hiponatremia, hipoalbuminemia, aumento de las transaminasas y creatininemia, y ocasionalmente albuminuria ${ }^{8,10-13,16,17,20,21,23,25-28}$. Las publicaciones que incluyen análisis de líquido cefalorraquídeo han mostrado un resultado alterado en 6 de 9 pacientes, con pleocitosis mononuclear en tres y polimorfonuclear (PMN) en tres $^{10,12,27}$. En estos casos, la cuantía de los leucocitos en el LCR no ha superado las 213 células por $\mathrm{mm}^{3}$.

El compromiso multisistémico secundario a la vasculitis generalizada es evidenciado en las autopsias, habiéndose documentado miocarditis, encefalitis, nefritis, neumonía intersticial y linfadenitis generalizada, entre $\operatorname{otros}^{10,16,17,20,24,25,27,28}$. La vasculitis es del tipo linfohistiocitario $^{11}$ o necrosante ${ }^{27}$. En una de las comunicaciones se destaca la ausencia de compromiso inflamatorio cerebral a pesar del compromiso neurológico grave, con convulsiones y coma ${ }^{28}$, lo que parece estar asociado a mayor letalidad en los primeros días ${ }^{30}$.

En la Tabla 1 se muestra la frecuencia de algunos de los signos y síntomas más habituales en algunas de las series publicadas.

Varias publicaciones destacan que es muy frecuente la falta de sospecha clínica de la etiología por Rickettsia, lo que sin lugar a dudas contribuye a una mala evolución de los pacientes e incluso a un desenlace fatal. Los diagnósticos clínicos iniciales en estos casos han sido fiebre amarilla $^{15,21}$, meningitis ${ }^{27}$, fiebre, petequias y cefalea ${ }^{16}$, meningococcemia ${ }^{12}$, sarampión ${ }^{19,27}$, dengue o dengue hemorrágico $^{12,13,23,26}$, leptospirosis ${ }^{10,12-14}$, encefalitis equina ${ }^{16}$, síndrome HELP en mujeres embarazadas ${ }^{17}$ y sepsis ${ }^{12}$.

Varios autores indican un predominio de la afectación y gravedad de la infección en niños ${ }^{13,14,27-29}$. Zavala-Castro y cols. indican en su serie que los niños fueron los únicos que presentaron afectación neurológica (convulsiones) y que en ellos se concentraron las muertes (3 de 9 pacientes), incluyendo un recién nacido de 9 días de vida ${ }^{13}$. Todas estas muertes se produjeron en las primeras 24 $\mathrm{h}$ de ingreso en el hospital. En ninguno de los niños se sospechó rickettsiosis, por lo que no recibieron la terapia apropiada $^{13}$. En otro brote de cuatro casos fatales, tres eran niños $^{28}$. Finalmente, en un brote que afectó a seis niños, se produjo el óbito en cinco de ellos ${ }^{9}$.

Como se ha mencionado, la mortalidad es elevada y es debida a falla multisistémica secundaria a la vasculitis generalizada. La infección por $R$. rickettsii es la rickettsiosis más grave, con tasas de letalidad fluctuantes entre 20 y $40 \%$ y hasta 95\%. En Costa Rica se comunicó la muerte de dos de nueve pacientes ${ }^{12}$, y de tres pacientes en dos meses en dos localidades rurales ${ }^{19}$. En Argentina fallecieron dos niños en un brote de $\operatorname{seis}^{27}$ y los cuatro casos $(100 \%)$ en el brote descrito por Padock y cols. ${ }^{28}$. En Colombia se comunicaron 62 muertes en el primer brote de 65 pacientes descrito por Patino y cols., en $1937^{21}$; dos muertes en el brote de los Córdobas ${ }^{23}$; cuatro muertes en un brote que afectó a 15 personas ${ }^{25}$ y cuatro fallecimientos en la serie de 20 pacientes de Hidalgo y cols. ${ }^{26}$. En Panamá, seis de los 10 casos comunicados en 10 años resultaron fatales ${ }^{16} \mathrm{y}$ se produjeron tres muertes en un brote familiar en $2011^{17}$. En México fallecieron dos de nueve $\operatorname{casos}^{12}$ y 10 de los 21 casos ocurridos durante un año ${ }^{14}$. En Brasil murieron ocho de 17 pacientes $^{8}$ y cuatro de seis pacientes ${ }^{7}$.

Concomitantemente a la afectación humana se ha comunicado afectación de animales domésticos. Así

Tabla 1. Principales signos y síntomas de la infección por Rickettsia rickettsii en series latinoamericanas

\begin{tabular}{|c|c|c|c|}
\hline Síntomas/signos & $\begin{array}{c}(n=15) \\
\text { Colombia } \\
\%\end{array}$ & $\begin{array}{c}(n=8) \\
\text { México } \\
\%\end{array}$ & $\begin{array}{c}(n=9) \\
\text { México } \\
\%\end{array}$ \\
\hline Fiebre & 100 & 100 & 100 \\
\hline Mialgias & 87 & NR & NR \\
\hline Náuseas & NR & 88 & NR \\
\hline Vómitos & 60 & NR & NR \\
\hline Artralgias & 60 & NR & NR \\
\hline Dolor abdominal & 53 & NR & NR \\
\hline Dolor retro orbicular & 33 & NR & NR \\
\hline Ictericia & 27 & NR & NR \\
\hline Exantema & 20 & 78 & 100 \\
\hline Edema periorbitario & NR & NR & 78 \\
\hline Síntomas neurológicos & NR & 78 & 89 \\
\hline Síntomas respiratorios & NR & 67 & NR \\
\hline Menores de 15 años & 40 & 88 & $9 / 100$ \\
\hline Letalidad & 27 & 38 & 22 \\
\hline Referencia & $\begin{array}{c}\text { Pacheco y cols. } \\
2008^{25}\end{array}$ & $\begin{array}{l}\text { Zavala-Castro y cols. } \\
\qquad 2008^{13}\end{array}$ & $\begin{array}{l}\text { Martínez-Medina y cols. } \\
\qquad 2007^{12}\end{array}$ \\
\hline
\end{tabular}

NR: No reportado 
Zavala-Castro y cols. comunican la muerte de un perro y una oveja de una familia en la que se diagnosticó un caso humano fatal ${ }^{11}$. En otra publicación se ha reportado la muerte de dos perros y la recuperación de un tercero con doxiciclina en un hogar donde hubo dos casos humanos fatales $^{24}$. También se ha documentado la infección por $R$. rickettsii en dos perros en Brasil, en este caso sin relación a la aparición de casos humanos ${ }^{31}$.

En cuanto a las secuelas, de siete supervivientes de una serie de nueve pacientes, cuatro curaron sin secuelas y tres con secuelas neurológicas del tipo de déficit motor con dificultad para la marcha, el habla, la deglución e indiferencia a los estímulos externos ${ }^{12}$. En otras series también se han comunicado secuelas neurológicas ${ }^{27}$.

El principal vector de $R$. rickettsii en Latinoamérica es Amblyomma cajennense, reportado en México ${ }^{2,11,13,14}$, Brasil $^{2,7,9,10}$, Panamá ${ }^{2,15,16}$, Argentina ${ }^{2,28}$, Costa Rica $^{2,20}$ y Colombia ${ }^{2,21,24}$. También se ha documentado su presencia en A. aurelatum en Brasil, Haemaphysalis leporispalustris en Costa Rica, Dermacentor nitens en Panamá, Amblyomma imitator y Rhipicephalus sanguineus en México².

\section{Rickettsia parkeri}

Esta especie fue descrita por primera vez en 1939 en garrapatas Amblyomma maculatum en E.U.A. y fue considerada no patogénica hasta el año 2004, en que se reconocieron los primeros casos humanos ${ }^{32,33}$. Varios casos que inicialmente fueron diagnosticados como fiebre de las Montañas Rocosas en E.U.A. posteriormente han sido diagnosticados como infección por R. parkeri ${ }^{34,35}$. Las características clínicas observadas en 12 pacientes y 92 niños diagnosticados en E.U.A. han sido resumidas en sendos artículos ${ }^{36,37}$. En Latinoamérica se han comunicado casos humanos en Uruguay, Argentina y Brasil ${ }^{38-39}$.

En Uruguay, se sospechó la implicación de $R$. parkeri de forma paralela a la descripción en E.U.A. como agente causal de las fiebres manchadas transmitidas por garrapatas. Así, se encontró $R$. parkeri en un ejemplar de $A$. triste retirado del cuero cabelludo en una paciente que desarrolló fiebre y exantema maculo-papular con linfadenopatía regional ${ }^{40}$. En ese país, los primeros casos de rickettsiosis tras picadura de garrapatas fueron atribuidos inicialmente a $R$. conorii y consistieron en la comunicación de tres y 12 pacientes respectivamente, que presentaron fiebre, lesión maculo-papular y/o escara en el sitio de la picadura asociada a linfadenopatía regional dolorosa y ocasional exantema ${ }^{41,42}$. Posteriormente, estos casos fueron atribuidos a $R$. parkeri al identificar su presencia en $A$. triste $^{40,43}$. También en Uruguay, otros tres casos han sido atribuidos a $R$. parkeri mediante serología utilizando antígenos purificados ${ }^{44}$. Recientemente se ha publicado un nuevo caso confirmado por RPC en España, en un viajero procedente de Uruguay que presentó fiebre, escara y exantema maculo-papular ${ }^{38}$.
En Argentina, el primer caso probable de infección por $R$. parkeri corresponde a un hombre que desarrolló fiebre, mialgias, exantema y una lesión papulo-erosiva asociada a linfadenopatía local en el sitio de la picadura de una garrapata $^{45}$. Posteriormente, se identificó $R$. parkeri mediante técnicas de RPC en $A$. triste recolectadas en la misma área geográfica ${ }^{46}$. Recientemente, se han comunicado nueve casos sospechosos entre 2004 y 2009, en dos de los cuales se pudo confirmar la presencia de $R$. parkeri por RPC de la escara ${ }^{39}$. Todos tenían antecedentes de picadura de garrapata durante actividades recreativas o laborales. Ocho de ellos presentaron escara de inoculación indolora de 1 a 1,5 cm de diámetro, fiebre de seis días de duración como promedio, exantema maculo-papular no prurítico, en extremidades y tronco, asociado frecuentemente a pústulas o vesículas y a veces a petequias. Además de las manifestaciones bien conocidas y comunes para todas las rickettsiosis, como cefalea, mialgias, artralgias, se observó en algunos casos odinofagia, diarrea, fotofobia e inyección conjuntival. Todos presentaron un curso relativamente benigno recuperándose tras la terapia con doxiciclina ${ }^{39}$. En ese país las picaduras por garrapatas se producen principalmente entre agosto y noviembre, época en que los ejemplares adultos son relativamente abundantes y buscan alimento ${ }^{47}$. En la Figura 3 se muestra un paciente con infección por $R$. parkeri, confirmada mediante RPC, en la que se observan signos de celulitis local con escara y exantema maculo-papular acompañante.

En Brasil, se han publicado dos pacientes afectados de una infección por un "nuevo agente", cuya secuencia molecular muestra un alto porcentaje de similitud con $R$. parkeri, $R$. africae y $R$. sibirica. El primer paciente procedía del bosque lluvioso Atlántico de Brasil, y presentó una picadura de garrapata en la espalda desarrollando 10 días más tarde fiebre, exantema macular, mialgias, artralgias y una escara en el sitio de la picadura. Tras la instauración de un tratamiento con doxiciclina se produjo una rápida mejoría ${ }^{48}$. El segundo caso fue picado por una garrapata mientras hacía actividades deportivas en una región semiárida de Bahía, y nueve días después desarrolló fiebre alta (hasta $40^{\circ} \mathrm{C}$ ), mialgias, edema y dolor en sitio de la picadura que evolucionó a escara y adenopatía regional dolorosa. Además desarrolló un exantema maculo-papular generalizado y lesiones ulceradas dolorosas en la mucosa oral. Los síntomas remitieron rápidamente tras el inicio de tratamiento con doxiciclina ${ }^{49}$.

El principal signo clínico que distingue la infección por $R$. parkeri de la fiebre de las Montañas Rocosas es la frecuente presencia de una escara en el sitio de la mordedura de garrapata, similar a la "tache noir" de la fiebre exantemática Mediterránea, y su curso clínico benigno. Considerando la conocida reactividad cruzada entre las diferentes especies de Rickettsia en los test serológicos, se estima que muchos de los casos diagnosticados como 
fiebre de las Montañas Rocosas, en particular aquellos que se han presentado con escara, probablemente correspondían a infecciones por $R$. parkeri ${ }^{36}$.

El principal vector de $R$. parkeri en Latinoamérica es $A$. triste $e^{40,43,46,50,51}$ aunque ha sido aislado también en A. tigrinum en Bolivia ${ }^{52}$. Considerando que $A$. triste es endémico en al menos 12 otros países de Latinoamérica, se estima que esta infección podría estar presente en la mayor parte de la región ${ }^{43}$. La elevada tasa de infección de la población de garrapatas del género Amblyomma con $R$. parkeri comparada con la de $R$. rickettsii en sus vectores sugiere que existe una gran cantidad de infecciones por este agente que no son adecuadamente identificadas ${ }^{53}$.

En la Tabla 2 se indican las principales diferencias clínicas entre $R$. rickettsii y $R$. parkeri, los dos agentes más frecuentemente identificados en la región.

\section{Rickettsia felis}

A pesar de que el vector reconocido de esta Rickettsia es la pulga del gato (Ctenocephalides felis), se la ha identificado en varios otros vectores ${ }^{54}$, incluidas las ga$\operatorname{rrapatas}^{55,56}$. Este hecho, junto a la importante emergencia de casos de infección humana y su amplia distribución en Latinoamérica, motivan que se incluya en esta revisión. Rickettsia felis ha sido identificada causando enfermedad en humanos en varios países de Europa, Africa, Australia y Nueva Zelanda ${ }^{57}$. En dos de las publicaciones de Europa se aprecia agregación de $\operatorname{casos}^{58,59}$ y también en una publicación de Australia ${ }^{60}$. En Latinoamérica se han comunicado numerosos casos en México ${ }^{61-63}$ y Brasil ${ }^{64,65}$. Los síntomas incluyen fiebre, cefalea, artralgias, mialgias, síntomas respiratorios y abdominales. El exantema se presenta en menos de $50 \%$ de los casos, como macular o maculo-papular pero no petequial. No se ha comunicado escara de inoculación. Los casos reportados en Europa y Australia han presentado un curso leve, con rápida recuperación, tanto con o sin terapia antimicrobiana, con la excepción de una niña en Australia que presentó compromiso de coagulación y edema pulmonar requiriendo una corta estadía en una unidad de cuidados intensivos ${ }^{60}$. A diferencia del curso mayoritariamente benigno observado en otros continentes, en Latinoamérica se ha descrito compromiso de sistema nervioso central en algunos pacientes, consistentes en pérdida auditiva, meningismo y coma ${ }^{63,64}$. No se han comunicado muertes por $R$. felis.

\section{Rickettsia massiliae}

El único caso adquirido en América hasta la fecha corresponde a una mujer diagnosticada en España, que adquirió la infección en Buenos Aires donde había extraído garrapatas a su perro. La paciente presentó fiebre, exantema maculo-papular purpúrico generalizado, que incluía palmas y plantas, efusión pleural y una escara en la pierna en la que se identificó $R$. massiliae por RPC ${ }^{66}$. Este agente ha sido encontrado en $R$. sanguineus en E.U.A. ${ }^{67}$ y en Argentina ${ }^{68}$.

Tabla 2.- Manifestaciones clínicas de las dos principales rickettsiosis de Latinoamérica

\begin{tabular}{|lll|}
\hline Manifestación clínica & Rickettsia rickettsii & Rickettsia parkeri \\
Fiebre & Sí & Sí \\
Mialgias, artralgias & Sí & Sí \\
Exantema & Sí (20-100\%) & Ocasional \\
Características del exantema & Maculo-papular o petequial & Macular o maculo-papular. Se han descrito vesículas, \\
pústulas y petequias
\end{tabular}




\section{Rickettsias identificadas en garrapatas presentes en Latinoamérica sin poder patógeno demostrado para humanos}

Las especies de Rickettsia y Candidatus a nuevas especies que se detallan a continuación hasta el momento no se han asociado con el desarrollo de manifestaciones clínicas. Sin embargo, se incluyen puesto que se debe considerar que pueden ser causa de enfermedad, al igual que ha sucedido con otras especies de Rickettsia a lo largo de la historia.

\section{Rickettsia bellii}

Esta especie ha sido encontrada en diversas especies de Amblyomma en Brasil y Argentina ${ }^{2,73}$ y más recientemente en El Salvador ${ }^{69}$.

\section{Candidatus rickettsia andeanae}

Este agente fue encontrado por primera vez en un $\mathrm{Am}$ blyomma spp. del norte de Perú ${ }^{70}$. Estudios filogenéticos mostraron que pertenece al grupo de las fiebres manchadas $^{71}$. Ha sido identificada posteriormente en $A$. parvum en Córdova, Argentina ${ }^{72}$, en A. maculatum en E.U.A. ${ }^{74}$ y recientemente en $A$. triste y $A$. tigrinum de zonas rurales en varias regiones de Chile ${ }^{75,76}$.

\section{Rickettsia sp. strain colombianensi}

Este nueva cepa de Rickettsia fue aislada recientemente en garrapatas A. dissimile, R. micropus y otros Amblyomma spp., recogidas sobre iguanas y vegetación en la región de Montería y Los Córdobas (Colombia) ${ }^{77}$.

\section{Conclusiones}

Las infecciones por Rickettsias transmitidas por garrapatas constituyen zoonosis emergentes de creciente reconocimiento e importancia en el mundo. Sin embargo, aún existe un importante sub-diagnóstico que puede llevar a un peor pronóstico de los enfermos. En este artículo se resumen las características clínicas de la infección por R. rickettsii, R. parkeri, R. felis y $R$. massiliae reportadas en Latinoamérica. También se indican rickettsias de reciente identificación en la región, de las que aún no se ha demostrado un rol patógeno para las personas.

Agradecimientos. A Rodrigo Angerami del Hospital of Clinics, State University of Campinas/UNICAMP. Health Department Surveillance, Campinas, São Paulo por la cesión de la Figura 1 y 2 correspondientes a exantemas en pacientes afectos de infección por Rickettsia rickettsii.

\section{Resumen}

Las rickettsiosis transmitidas por garrapatas son infecciones potencialmente letales, que en Latinoamérica tienen carácter emergente y re-emergente. Hasta hace escasos años, la única rickettsiosis transmitida por garrapatas era causada por Rickettsia rickettsii, pero en la actualidad existen otras especies como R. parkeri y $R$. massiliae que están provocando enfermedad en humanos en la región. Por otro lado, se están describiendo candidatos a nuevas especies de Rickettsia, que aunque no han probado su patogenicidad deben considerarse como potencialmente patógenos. Dado que el diagnóstico microbiológico puede tardar días o semanas, resulta fundamental una alta sospecha clínica y la instauración precoz de un tratamiento adecuado. En esta revisión se detalla la distribución y principales manifestaciones clínicas de las rickettsiosis transmitidas por garrapatas en Latinoamérica. Se ha incluido una sección sobre la infección por $R$. felis, por haberse encontrado esta especie en garrapatas, y no haberse aclarado el papel de este vector en su ciclo epidemiológico.

\section{Referencias bibliográficas}

1.- Hechemy K E, Oteo J A, Silverman D, Raoult D, Blanco J R. A Century of Rickettsiology: Emerging, reemerging rickettsioses, clinical, epidemiologic, and molecular diagnostic aspects and emerging veterinary rickettsioses. An overview. Ann NY Acad Sci 2006; 1077: 1-14.

2.- Labruna M, Mattar S, Nava S, Bermúdez S, Venzal J M, Dolz G, et al. Rickettsiosis in Latin America, Caribean, Spain and Portugal. Rev MVZ Cordoba 2011; 16: 2435-57.

3.- Oteo J A, Portillo A. Tick-borne rickettsiosis in Europe. Ticks and tick-borne Dis 2012; 3: 270-7.

4.- Parola P, Paddock C D, Socolovschi C D, Labruna MB, Mediannikov O, Kernif T, et al.
Update on tick-borne rickettsioses around the world: a geographic approach. Clin Microb Rev 2013; 26: 657-702.

5.- Walker D H. Rickettsia rickettsii and other spotted fever Group Rickettsiae (Rocky Mountain spotted fever and other spotted fevers). In: Mandell, Douglas, and Bennett's Principles and Practice of Infectious Diseases, Mandell GL; Bennett JE; Dolin R. (Eds.) 7th Ed. Churchill Livingstone Elsevier. Philadelphia, USA. 2010; pp. 2499-508.

6.- Gonçalves A J R, Lopes P F A, Melo J C P, Pereira A A, Pinto A M M, Lazera M S, et al. Rickettsioses-a propósito de quatro casos diagnosticados no Rio de Janeiro de febre maculosa brasileira. F Méd (BR) 1981; 82: 127-34.
7.- Sexton D J, Muniz M, Corey G R, Breitschwerdt E B, Hegarty B C, Dumler S, et al. Brazilian spotted fever in Espirito Santo, Brazil: description of a focus of infection in a new endemic region. Am J Trop Med Hyg 1993; 49: 222-6.

8.- De Lemos E R, Alvarenga F B, Cintra M L, Ramos M C, Paddock C D, Ferebee T L, et al. Spotted fever in Brazil: a seroepidemiological study and description of clinical cases in an endemic area in the state of São Paulo. Am J Trop Med Hyg 2001; 65: 329-34.

9.- Galvão M A, Dumler J S, Mafra C L, Calic S B, Chamone C B, Cesarino Filho G, et al. Fatal spotted fever rickettsiosis, Minas Gerais, Brazil. Emerg Infect Dis 2003; 9: 1402-5.

10.- Lamas C, Favacho A, Rozental T, Bóia M N, 
Kirsten A H, Guterres A, et al. Characterization of Rickettsia rickettsii in a case of fatal Brazilian spotted fever in the city of Rio de Janeiro, Brazil. Braz J Infect Dis 2008; 12: 149-51.

11.- Zavala-Castro J E, Zavala-Velázquez J E, Walker D H, Ruiz Arcila E E, Laviada-Molina $\mathrm{H}$, Olano J P, et al. Fatal human infection with Rickettsia rickettsii, Yucatán, Mexico. Emerg Infect Dis 2006; 12: 672-4.

12.- Martínez-Medina M A, Álvarez-Hernández G, Rojas-Guerra M G, Padilla-Zamudio J G. Fiebre manchada de las Montañas Rocosas en niños: consideraciones clínicas y epidemiológicas. Gac Méd Méx 2007; 143: 137-40.

13.- Zavala-Castro J E, Dzul-Rosado K R, León J J, Walker D H, Zavala-Velázquez J E. An increase in human cases of spotted fever rickettsiosis in Yucatan, Mexico, involving children. Am J Trop Med Hyg 2008; 79: 907-10.

14.- Álvarez-Hernández G. La fiebre manchada de las Montañas Rocosas, una epidemia olvidada. Salud Pública de México 2010; 52: 1-3.

15.- Rodaniche EC, Rodaniche A. Spotted fever in Panama. Isolation of the etiologic agent from a fatal case. Am J Trop Med Hyg 1950; 30: 511-7.

16.- Estripeaut D, Aramburú M G, Sáez-Llorens X, Thompson H A, Dasch G A, Paddock C D, et al. Rocky mountain spotted fever, Panama. Emerg Infec Dis 2007; 13: 1763-5.

17.- Tribaldos $\mathrm{M}$, Zaldivar $\mathrm{M}$, Bermúdez $\mathrm{S}$, Samudio F, Mendoza Y, Martínez A A, et al. Rocky Mountain spotted fever in Panama: a cluster description. J Infect Dev Ctries 2011; 5: 737-41.

18.- Fuentes L G. Primer caso de fiebre de las Montañas Rocosas en Costa Rica, América Central. Rev Latinoam Microbiol 1979; 21: 167-72.

19.- Hun L, Herrero L, Fuentes L, Vargas M. Tres nuevos casos de fiebres manchadas de las Montañas Rocosas en Costa Rica. Rev Costarricense Ciencias Médicas 1991; 12: 51-6.

20.- Hun-Opfer L. Revisión. Las fiebres manchadas y su importancia en Costa Rica. Acta Médica Costarricense 2008; 50: 77-86.

21.- Patino L, Afanador A, Paul J H. A spotted fever in Tobía, Colombia. Am J Trop Med Hyg 1937; 17: 639-53.

22.- Acosta J, Urquijo L, Díaz A, Sepúlveda M, Mantilla G, Heredia D, et al. Brote de rickettsiosis en Necoli, Antioquía, febreromarzo de 2006. Inf Quinc Epidemiol Nac 2006; 11: 177-92.

23.- Hidalgo M, Lizarazo D, Ovalle M, Castañeda E, Heredia D, Zambrano P, et al. Brote de rickettsiosis en Los Córdobas, departamento de Córdoba, febrero-marzo 2007. Inf Quinc Epidemiol Nac 2007; 12: 367-78.

24.- Hidalgo M, Orejuela L, Fuya P, Carrillo P, Hernández J, Parra E, et al. Rocky Mountain spotted fever, Colombia. Emerg Infect Dis 2007; 13: $1058-60$
25.- Pacheco O, Giraldo M, Hidalgo M, Galeano A, Echeverri I, Eccheverría L, et al. Estudio de brote febril hemorrágico en el corregimiento de Alto Mulatos-Distrito Especial Portuario de Turbo, Antioquía, enero de 2008. Inf Quinc Epidemiol Nac 2008; 13: 145-60.

26.- Hidalgo M, Miranda J, Heredia D, Zambrano P, Vesga J F, Lizarazo D, et al. Outbreak of Rocky Mountain spotted fever in Córdoba, Colombia. Mem Osv Cruz 2011; 106: 117-8.

27.- Ripoll C M, Remondegui C E, Ordoñez G, Arazamendi R, Fusaro H, Hyman M J, et al. Evidence of rickettsial spotted fever and ehrlichial infections in a subtropical territory of Jujuy, Argentina. Am J Trop Med Hyg 1999; 61: 350-4

28.- Paddock C D, Fernández S, Echenique G A, Sumner J W, Reeves W K, Zaki S R, et al. Rocky Mountain spotted fever in Argentina. Am J Trop Med Hyg 2008; 78: 687-92.

29.- Galvao M A, Dumler J S, Mafra C L, Calic S B, Chamone C B, Filho G C, et al. Fatal spotted fever rickettsiosis, Minas Gerais, Brazil. Emerg Infect Dis 2003; 9: 1402-5.

30.- Valbuena G, Bradford W, Walker D H. Expression analysis of the T-cell-targeting chemokines CXCL9 and CXCL10 in mice and humans with endothelial infections caused by rickettsiae of the spotted fever group. Am J Pathol 2003; 163: 1357-69.

31.- Labruna M B, Kamakura O, Moraes-Filho J, Horta M C, Pacheco R C. Rocky Mountain spotted fever in dogs, Brazil. Emerg Infect Dis 2009; 15: 458-60.

32.- Paddock C D, Summer J W, Comer J A, Zaki S R, Goldsmith C S, Goddard J, et al. Rickettsia parkeri: A newly recognized cause of spotted fever rickettsiosis in the United States. Clin Infect Dis 2004; 38: 805-11.

33.- Paddock C D. Rickettsia parkeri as a paradigm for multiple causes of tick-borne spotted fever in the western hemisphere. Ann NY Acad Sci 2005; 1063: 315-26.

34.- Raoult D, Paddock C D. Rickettsia parkeri and other spotted fever infections in the United States. N Engl J Med 2005; 353: 626-7.

35.- Whitman T J, Richards A L, Paddock C D, Tamminga C L, Sniezek P J, Jiang J, et al. Rickettsia parkeri infection after tick bite, Virginia. Emerg Infect Dis 2007; 13: 334-6.

36.- Paddock C D, Finley R W, Wright C S, Robinson H N, Schrodt B J, Lane C C, et al. Rickettsia parkeri rickettsiosis and its clinical distinction from Rocky Mountain spotted fever. Clin Infect Dis 2008; 47: 1188-96.

37.- Buckingham S C, Marshall G S, Schutze G E, Woods C R, Jackson M A, Petterson L E R, et al. Clinical and laboratory features, hospital course, and outcome of Rocky Mountain spotted fever in children. J Pediatrics 2007; 150: 180-4.

38.- Portillo A, García-García C, Sanz M M,
Santibáñez S, Venzal J M, Oteo J A. A Confirmed case of Rickettsia parkeri infection in a traveler from Uruguay. Am J Trop Med Hyg 2013; 89: 1203-5.

39.- Romer Y, Seijo AC, Crudo F, Nicholson W L, Varela-Stokes A, Lash RR et al. Rickettsia parkeri rickettsiosis, Argentina. Emerg Infect Dis 2011; 17: 1169-73.

40.- Venzal J M, Portillo A, Estrada-Pena A, Castro O, Cabrera P A, Oteo JA. Rickettsia parkeri in Amblyomma triste from Uruguay. Emerg Infect Dis 2004; 10: 1493-5.

41.- Conti-Díaz I A, Rubio I, Somma Moreira R, Pérez Dormida G. Rickettsiosis cutáneoganglionar por Rickettsia conorii en el Uruguay. Rev Inst Med Trop S Paulo 1990; 32: 313-8.

42.- Conti-Díaz I A. Rickettsiosis por Rickettsia conorii (fiebre botonosa del Mediterráneo o fiebre de Marsella). Estado actual en Uruguay. Rev Med Uruguay 2001; 17: 119-24.

43.- Pacheco R C, Venzal J M, Richtzenhain L J, Labruna M B. Rickettsia parkeri in Uruguay. Emerg Inf Dis 2006; 12: 1804-5.

44.- Conti-Díaz I A, Moraes-Filho J, Pacheco R C, Labruna M B. Serological evidence of Rickettsia parkeri as etiological agent of rickettsiosis in Uruguay. Rev Inst Med Trop Sao Paulo 2009; 51: 337-9.

45.- Seijo A, Picollo M, Nicholson W, Paddock C. Fiebre manchada por rickettsias en el Delta del Paraná: una enfermedad emergente. Medicina (B Aires) 2007; 67: 723-6.

46.- Nava S, Elshenawy Y, Eremeeva M E, Sumner J W, Mastropaolo M, Paddock C D. Rickettsia parkeri in Argentina. Emerg Infec Dis 2008; 14: 1894-7.

47.- Nava S, Mangold A J, Mastropaolo M, Venzal J M, Fracassi N, Guglielmone A A. Seasonal dynamics and hosts of Amblyomma triste (Acari: Ixodidae) in Argentina. Vet Parasitol 2011; 181: 301-8.

48.- Spolidorio M G, Labruna M B, Mantovani E, Brandao P E, Richtzenhain L J, Yoshinari N H. Novel spotted fever group rickettsiosis, Brazil. Emerg Infect Dis 2010; 16: 521-3.

49.- Silva N, Eremeeva M E, Rozental T, Ribeiro G S, Paddock C D, Ramos E A G, et al. Eschar-associated spotted fever rickettsiosis, Bahia, Brazil. Emerg Infect Dis $2011 ; 17: 275-8$

50.- Silveira I, Pacheco R C, Szabó M P, Ramos H G, Labruna MB. Rickettsia parkeriin Brazil. Emerg Infect Dis 2007; 13: 1111-3.

51.- Venzal J M, Nava S. El género Rickettsia como agente de zoonosis en el Cono Sur de Sudamérica. Rev Med Urug 2011; 27: 98-106.

52.- Conte V, Parrilla G, de Meneghi D, Tomassone L. Prevalence of Rickettsia infection in dogs and detection of Rickettsia parkeri in Amblyoma tigrinum ticks in Cochabamba Department, Bolivia. Book of Proceedings, VI International Conference on 
ticks and tick-borne pathogens. Buenos Aires, Argentina, 2008, page 076.

53.- Labruna M B. Ecology of Rickettsia in South America. Ann N Y Acad Sci 2009; 1166 : 156-66.

54.- Mediannikov O, Audoly G, Diatta G, Trape J F, Raoult D. New Rickettsia sp. in tsetse flies from Senegal. Comp Immunol Microbiol Infect Dis 2012; 35: 145-50

55.- Gargill A, Palomar A, Midill K, Portillo A, Kar S, Oteo J A. Rickettsia species in ticks removed from humans in Istambul, Turkey. Vect Borne Infect Dis 2012; 12: 938-41.

56.- Abarca K, López J, Acosta-Jamett G, Martínez-Valdebenito C. Rickettsia felis in Rhipicephalus sanguineus from two distant Chilean cities. Vect Borne Infect Dis 2013; 13: 607-9.

57.- Pérez-Osorio CE, Zavala-Velázquez J E, Arias León J J, Zavala-Castro J E. Rickettsia felis as emergent global threat for humans. Emerg Infect Dis 2008; 14: 1019-23.

58.- Oteo J A, Portillo A, Santibáñez S, Blanco J R, Pérez-Martínez L, Ibarra V. Cluster of cases of human Rickettsia felis infection from Southern Europe (Spain) diagnosed by PCR. J Clin Microbiol 2006; 44: 2669-71.

59.- Richter J, Fournier PE, Petridou J, Häussinger D, Raoult D. Rickettsia felis infection acquired in Europe and documented by polymerase chain reaction. Emerg Infect Dis 2002; 8: 207-8.

60.- Williams M, Izzard L, Graves S R, Stenos J, Kelly JJ. First probable Australian cases of human infection with Rickettsia felis (cat-flea thypus), Australia. M J A 2011; 194: 41-3.

61.- Zavala-Velázquez J E, Ruiz-Sosa J A, Sánchez-Elias R A, Becerra-Carmona G, Walker D H. Rickettsia felis rickettsiosis in Yucatán. Lancet 2000; 356: 1079-80.

62.- Zavala-Velázquez J, Laviada-Molina H,
Zavala-Castro J, Pérez-Osorio C, BecerraCarmona G, Ruiz-Sosa JA, et al. Rickettsia felis, the agent of an emerging infectious disease: Report of a new case in Mexico. Arch Med Res 2006; 37: 419-22.

63.- Zavala-Castro J E, Zavala-Velázquez J, Walker D, Pérez-Osorio J, Peniche-Lara G. Severe human infection with Rickettsia felis associated with hepatitis in Yucatan, Mexico. Int J Med Microbiol 2009; 299: 529-33.

64.- Galvao M A, Mafra C, Chamone C B, Calic S B, Zavala-Velásquez J E, Walker D H. Clinical and laboratorial evidence of Rickettsia felis infections in LatinAmerica. Revista Sociedade Brasileira Medicina Tropical 2004; 37: 238-40.

65.- Galvao M A, Zavala-Velázquez J E, Zavala-Castro J E, Mafra C L, Calic S B, Walker D H. Rickettsia felis in the Americas. Ann N Y Acad Sci 2006; 1078: 156-8.

66.- García- García J C, Portillo A, Núñez M J, Santibáñez S, Castro B, Oteo J A. Case report: a patient from Argentina infected with Rickettsia massiliae. Am J Trop Hyg 2010; 82: 691-2.

67.- Eremeeva M E, Bosserman E A, Demma L J, Zambrano M L, Blau D M, Dasch G A. Isolation and identification of Rickettsia massiliae from Rhipicephalus sanguineus ticks collected in Arizona. Appl Environ Microbiol 2006; 72: 5569-77.

68.- Cicuttin G L, Rodríguez M, Jado I, Anda P. Primera detección de Rickettsia massiliae en la Ciudad de Buenos Aires. Resultados preliminares. Rev Arg Zoonosis 2004; 1: 8-10.

69.- Barbieri A R, Romero L, Labruna M B. Rickettsia bellii infecting Amblyomma sabanerae ticks in El Salvador. Pathog Glob Health 2012; 106: 188-9.

70.- Blair P J, Jiang J, Schoeler G B, Moron C, Anaya E, Céspedes M, et al. Characterization of spotted fever group Rickettsiae in fleas and tick specimens from Northern Peru. J Clin Microbiol 2004; 42: 4961-7.

71- Jiang J, Blair P J, Vidal F, Moron C, Céspedes M, Anaya E, et al. Phylogenetic analysis of a novel molecular isolate of spotted fever group Rickettsiae from Northern Peru. Ann N Y Acad Sci 2005; 1063: 337-42.

72.- Pacheco R C, Moraes-Filho J, Nava S, Brandão P E, Richtzenhain L J, Labruna M B. Detection of a novel spotted fever group rickettsia in Amblyomma parvum ticks (Acari: Ixodidae) from Argentina. Exp Appl Acarol 2007; 43: 63-71.

73.- Tomassone L, Núñez P, Ceballos LA, Gurtler R E, Kitron U, Farber M. Detection of "Candidatus Rickettsia sp. strain Argentina" and Rickettsia bellii in Amblyomma ticks (Acari: Ixodidae) from Northern Argentina. Exp Appl Acarol 2010; 52: 93-100.

74.- Paddock C D, Fournier P E, Summer J W, Goddard J, Elshenawy Y, Metcalfe M G, et al. Isolation of Rickettsia parkeri and identification of a novel spotted fever group Rickettsia sp. from Gulf Coast ticks (Amblyomma maculatum) in the United States. Appl Environ Microbiol 2010; 76: 2689-96.

75.- Abarca K, López J, Acosta-Jamett G, Lepe P, Soares J F, Labruna M B. A third Amblyomma specie and the first tick-borne Rickettsia in Chile. J Med Entomol 2012; 49: 219-22.

76.- Abarca K, López J, Acosta-Jamett G, Martínez-Valdebenito C. Identificación de Rickettsia andeanae en dos regiones de Chile. Rev Chilena Infectol 2013; 30: 388-94.

77.- Miranda J, Portillo A, Oteo J A, Mattar S. Rickettsia sp. strain colombianensi (Rickettsiales: Rickettsiaceae): a new proposed Rickettsia detected in Amblyomma dissimile (Acari: Ixodidae) from iguanas and free larvae ticks from vegetation. J Med Entomol 2012; 49: 960-5. 